\title{
3-Substituted isocyanopyridines as mildly convertible isocyanides
}

\author{
Mathias Elsocht ${ }^{1}$, Karlijn Hollanders ${ }^{1}$, Olivier Van der Poorten ${ }^{1}$, Mouhamad Jida ${ }^{2}$, Bert Maes ${ }^{3}$, Steven Ballet ${ }^{1}$
}

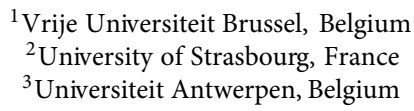

https://doi.org/10.17952/35EPS.2018.118

\section{Introduction}

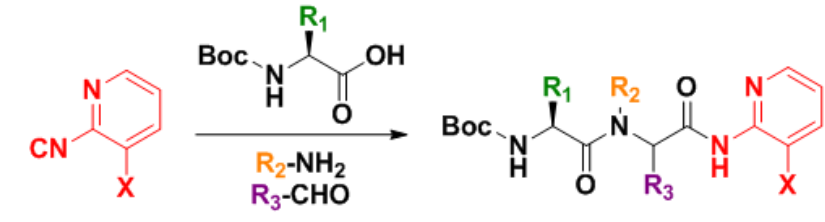

$\mathrm{X}=\mathrm{Br}, \mathrm{Cl}, \mathrm{OMe}$

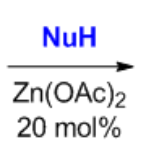

$20 \mathrm{~mol} \%$

Di- and Tripeptides synthesis

Scheme 1: Synthesis of Ugi products containing a directing group followed by a Zinc catalysed transamidation reaction.

Multicomponentreactions (MCRs) have become a powerful tool to generate peptidomimetics since they are easy to use and give great structural diversity. More specifically, the Ugi reaction forms an $\alpha$-acylaminocarboxamide utilizing an aldehyde, an amine, a carboxylic acid, and an isocyanide. Such dipeptide-like structures have found great application, often in combination with post-condensation modifications. The downside of this reaction is the formation of an amide bond, derived from the isocyanide, at the C-terminus of the product, which cannot be cleaved under mild reaction conditions. This limitation can be circumvented by the use of convertible isocyanides that allow the chemoselective transformation of this amide bond after the MCR.[1] Throughout the years, different convertible isocyanides have been developed all with their advantages and restrictions. For example, the isocyanide is not highly accessible or even unstable, or the amide cleavage requires acidic/basic conditions which in multistep procedures might result in the undesired cleavage of protecting groups. With this in mind, we set out to develop a convertible isocyanide that can be cleaved, under neutral conditions, using a $\mathrm{Zn}$ catalysed nicotinate-directed transamidation(Figure 1), a previously developed strategy by our groups.[2] Hence, the synthesis of different 3-substituted-2-isocyanopyridines was pursued, which were subsequently subjected to Ugi-4C conditions. These Ugi products were in turn used in the zinc catalysed transamidation reaction. It was demonstrated that, for all three directing groups, the amide bond at the C-terminus could easily and selectively be cleaved. As a proof of concept, we demonstrated the methodology in the synthesis of a previously published constrained dipeptide, which was obtained through Ugi-post-condensationmodifications. [3]

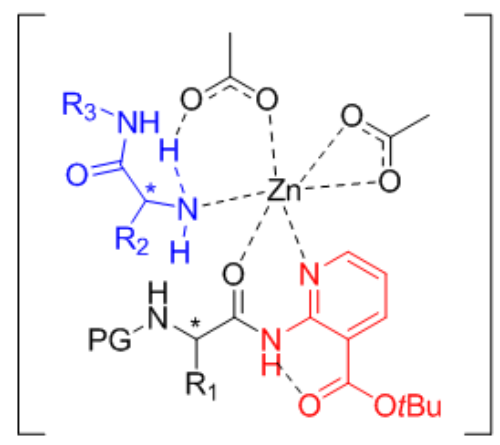

Figure 1: Starting complex in the Zn-catalysed transamidation reaction.

\section{Results and Discussion}

Synthesis of isocyanides 3 was achieved through the formylation and dehydration of the correspondingamines 1. The dehydration was performed using $\mathrm{PhPO} 2 \mathrm{Cl} 2$, which is a green variant of $\mathrm{POCl} 3$ and more suitable for the synthesis of isocyanides of pyridine-based formamides 2.[5] This reagent gave access to all three desired isocyanides in good yield. 
Table 1: Synthesis of 3-substituted-2-isocyanopyridines 3 via the formylation/dehydration sequence.

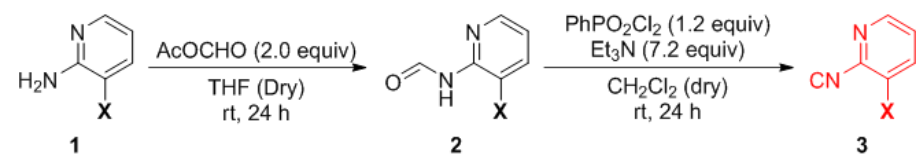

\begin{tabular}{cccccc}
\hline Entry & $\mathbf{X}$ & $\mathbf{2}$ & Yield of 2 (\%) & 3 & Yield of 3 (\%) \\
\hline 1 & $\mathrm{Br}$ & 2a & $>99$ & 3a & 53 \\
2 & $\mathrm{Cl}$ & 2b & $>99$ & 3b & 63 \\
3 & $\mathrm{OMe}$ & 2c & $>99$ & 3c & 61 \\
\hline
\end{tabular}

Subsequently, the different isocyanides 3 were used in an Ugi four component reaction towards 4. Here, isovaleraldehyde and propylamine were stirred in TFE for 2 hours prior to the addition of the N-Boc protected phenylalanine and the isocyanides 3, yielding the desired Ugi products 4 in good yield. In a next step, the Ugi products 4, equipped with the directing groups, were used in the Zn-catalysed transamidation reaction. Comparable ${ }^{1} \mathrm{H}-\mathrm{NMR}$ yields were obtained for all three directing groups which let us belief that all three directing groups are equally efficient in the transamidation reaction. Nevertheless, as the chlorinoated directing group gave the highest yield in the Ugi reaction, we selected $3 b$ for the synthesis of aminotriazoloazepinone 6 .

Table 2: Synthesis of directing group containing Ugi product 4 and evaluation of directing groups in the $\mathrm{Zn}$ catalysed transamidation reaction

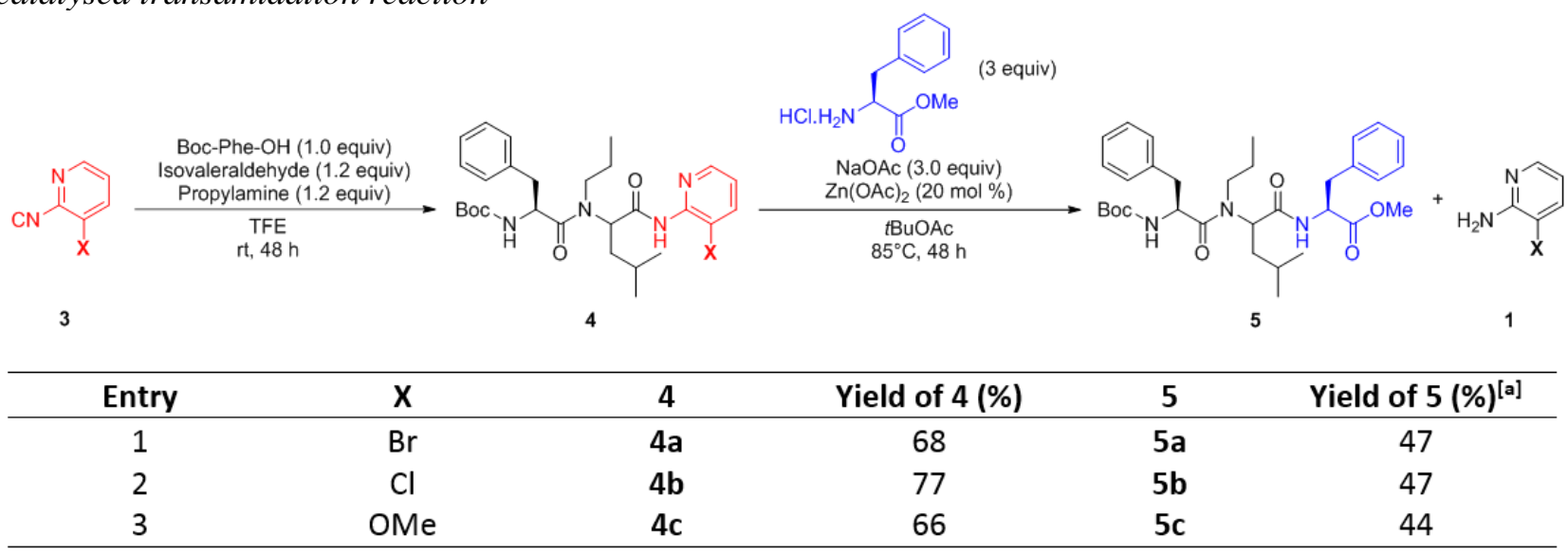

[a] ${ }^{1} \mathrm{H}$ NMR yield using 1,3,5-trimethoxybenzene as internal standard, corrected to a mass balance of $100 \%$.

The 3-chloro-2-isocyanopyridine $3 \mathrm{~b}$ was used in the synthesis of aminotriazoloazepinone-containing dipeptides 6, following the one-pot Ugi-Huisgen cyclization.[3] Afterwards, a $\mathrm{Zn}(\mathrm{OAc})_{2}$-mediated hydrolysis was carried out towards compound 7 , which was coupled, without intermediate purification, on solid-support by a standard coupling method. Final cleavage yielded the desired tripeptides 9a-b. Hence, the convertible isocyanide $3 \mathrm{~b}$ made it possible to generate a labile amide at the C-terminus of the Ugi product, which allowed to generate, after hydrolysis, a SPPS-compatible dipeptide mimetic. 


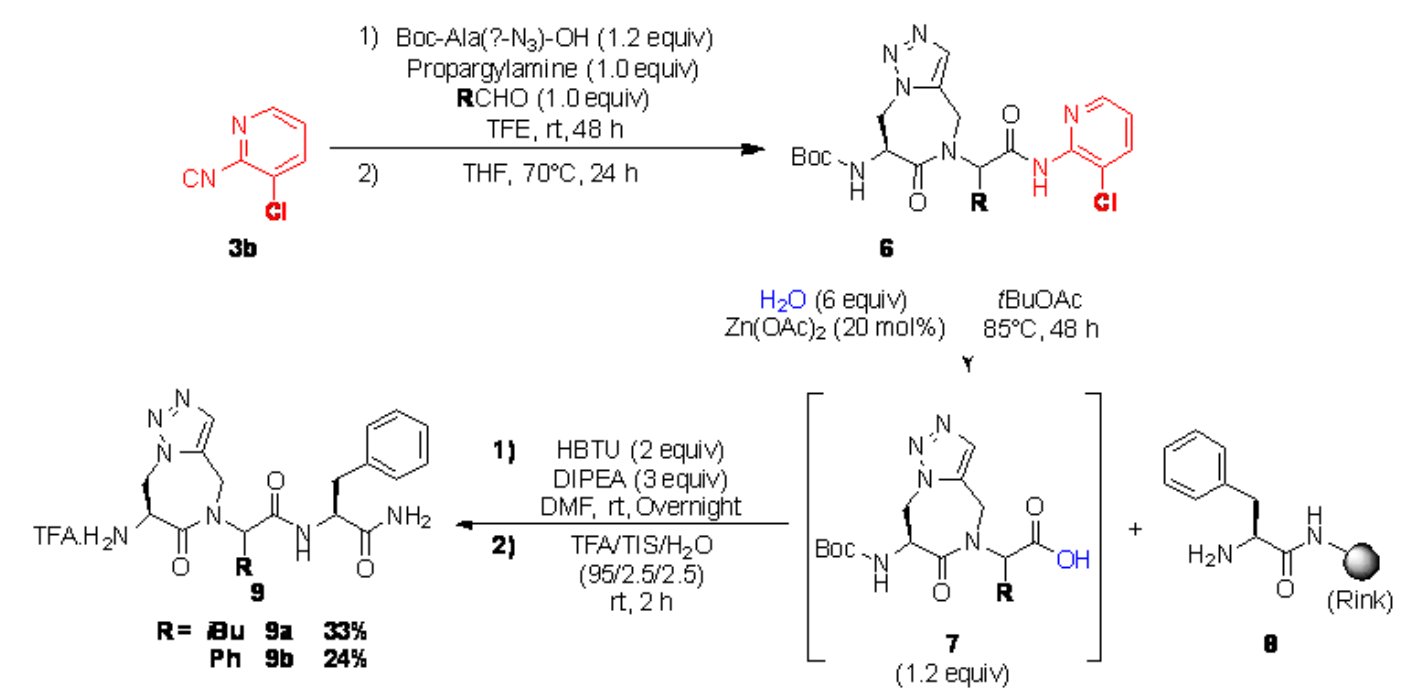

Scheme 2: Synthesis of aminotriazoloazepinone-containing dipeptides 6 followed by the anchoring on solidsupport in a two-step procedure: hydrolysis - coupling.

\section{Conclusion}

Three new convertible isocyanides were developed that allow a C-terminal amide cleavage of Ugi products, under mild conditions, yielding di- and tripeptides. The practical utility of these cleavable isocyanides was demonstrated through the synthesis of a constrained dipeptide scaffold which was anchored on solid support after cleavage of the amide group utilizing a $\mathrm{Zn}$-catalyzed reaction.

\section{Acknowledgments}

$\mathrm{ME}$ and SB are grateful to the Doctoral school (VUB) for the financial support and acknowledge the organizing committee of 35th EPS for the registration bursary.

\section{References}

[1] M. Oikawa et al, Synlett. 24 (2013) 2014-2018. doi:10.1055/s-0033-1338967.

[2] C.C.D. Wybon et al, ACS Catalysis. 8 (2018) 203-218. doi:10.1021/acscatal.7b02599.

[3] T.M.A. Barlow et al, Org. Biomol. Chem. 12 (2014) 6986-6989. doi:10.1039/C4OB01381F .

[4] G. Kobayashi et al, Synthesis. 2011 (2011) 3225-3234. doi:10.1055/s-0030-1260211. 